PREPARED FOR THE U.S. DEPARTMENT OF ENERGY, UNDER CONTRACT DE-AC02-76CH03073

PPPL-3477

PPPL-3477

UC-70

Signatures of Mode Conversion and Kinetic Alfvén Waves at the Magnetopause

by

Jay R. Johnson and C.Z. Cheng

July 2000

$=$ PPPL

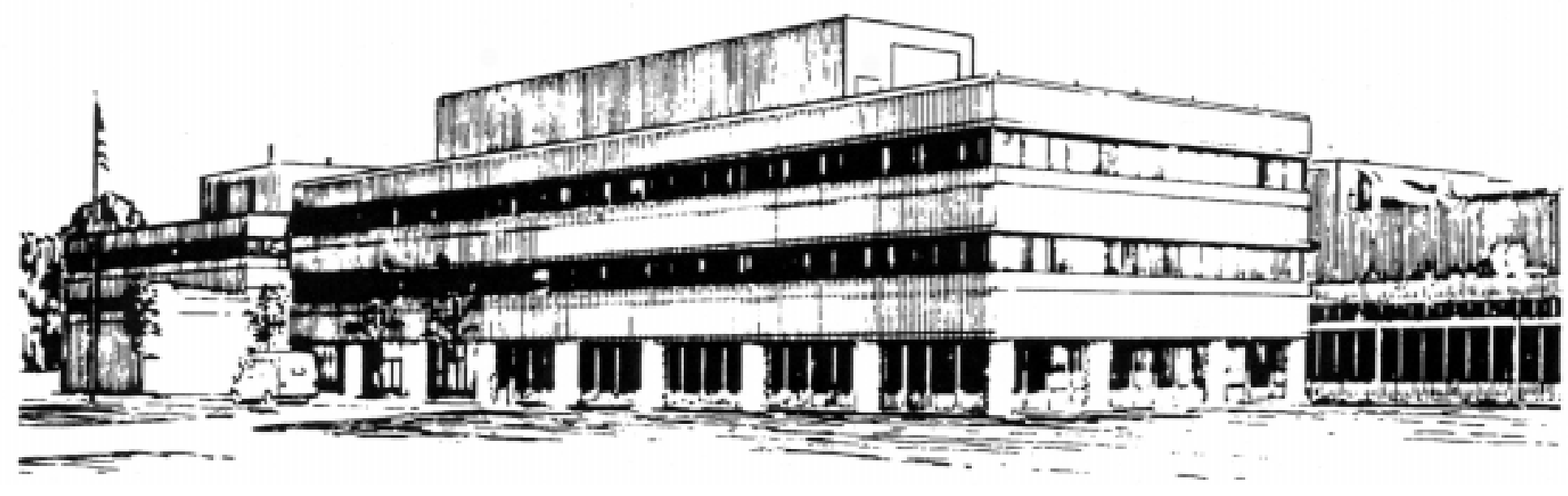

PRINCETON PLASMA PHYSICS LABORATORY PRINCETON UNIVERSITY, PRINCETON, NEW JERSEY 


\section{PPPL Reports Disclaimer}

This report was prepared as an account of work sponsored by an agency of the United States Government. Neither the United States Government nor any agency thereof, nor any of their employees, makes any warranty, express or implied, or assumes any legal liability or responsibility for the accuracy, completeness, or usefulness of any information, apparatus, product, or process disclosed, or represents that its use would not infringe privately owned rights. Reference herein to any specific commercial product, process, or service by trade name, trademark, manufacturer, or otherwise, does not necessarily constitute or imply its endorsement, recommendation, or favoring by the United States Government or any agency thereof. The views and opinions of authors expressed herein do not necessarily state or reflect those of the United States Government or any agency thereof.

\section{Availability}

This report is posted on the U.S. Department of Energy's Princeton Plasma Physics Laboratory Publications and Reports web site in Calendar Year 2000. The home page for PPPL Reports and Publications is: http://www.pppl.gov/pub_report/

DOE and DOE Contractors can obtain copies of this report from:

U.S. Department of Energy

Office of Scientific and Technical Information

DOE Technical Information Services (DTIS)

P.O. Box 62

Oak Ridge, TN 37831

Telephone: (865) 576-8401

Fax: (865) 576-5728

Email: reports@adonis.osti.gov

This report is available to the general public from:

National Technical Information Service

U.S. Department of Commerce

5285 Port Royal Road

Springfield, VA 22161

Telephone: $1-800-553-6847$ or

(703) $605-6000$

Fax: (703) 321-8547

Internet: http://www.ntis.gov/ordering.htm 
Submitted to Geophysical Research Letters, 2000.

\title{
Signatures of Mode Conversion and Kinetic Alfvén Waves at the Magnetopause
}

\author{
Jay R. Johnson and C. Z. Cheng \\ Princeton University, Plasma Physics Laboratory, Princeton, NJ
}

\begin{abstract}
It has been suggested that resonant mode conversion of compressional MHD waves into kinetic Alfvén waves at the magnetopause can explain the abrupt transition in wave polarization from compressional to transverse commonly observed during magnetopause crossings [Johnson and Cheng, 1997b]. We analyze magnetic field data for magnetopause crossings as a function of magnetic shear angle (defined as the angle between the magnetic fields in the magnetosheath and magnetosphere) and compare with the theory of resonant mode conversion. The data suggest that amplification in the transverse magnetic field component at the magnetopause is not significant up to a threshold magnetic shear angle. Above the threshold angle significant amplification results, but with weak dependence on magnetic shear angle. Waves with higher frequency are less amplified and have a higher threshold angle. These observations are qualitatively consistent with theoretical results obtained from the kinetic-fluid wave equations.
\end{abstract}

\section{Introduction}

Ultra-low frequency (ULF) waves (with frequencies below $500 \mathrm{mHz}$ ) dominate the spectrum of nearly every magnetopause crossing [Rezeau et al., 1993; Song, 1994; Song et al., 1993; Phan and Paschmann, 1996, and references therein]. It has been suggested that these waves are associated mode conversion of MHD waves in the magnetosheath to kinetic Alfvén waves at the magnetopause which occurs near a field line resonance location Lee et al. [1994]; Belmont et al. [1995]. The mode conversion process can explain (a) a shift in wave polarization at the magnetopause and (b) the amplification of the transverse magnetic field component by an order of magnitude [Johnson and Cheng, 1997b]. However, comparative studies between theory and observation have not been performed. In this work, we present evidence that ULF waves at the magnetopause are the result of a mode conversion process which transforms compressional MHD waves that originate in the magnetosheath into transverse shear/kinetic Alfvén waves at the magnetopause. Based on a data survey of ISEE1, ISEE2, and WIND magnetopause crossings, we examined the dependence of the mode conversion process on the magnetic shear angle (defined to be the angle between the magnetic field in the magnetosheath and magnetosphere on each side of the magnetopause). The results of the survey compare favorably with theoretical predictions based on the model developed by Johnson and Cheng [1997b].

\section{Magnetosheath/Magnetopause Wave Ac- tivity and Magnetic Shear}

The most striking wave feature associated with magnetopause crossings is the sharp transition in the polarization of low frequency wave power from compressional $\left(\delta B_{\|} \geq \delta B_{\perp}\right)$ in the magnetosheath to transverse $\left(\delta B_{\perp} \gg \delta B_{\|}\right)$at the magnetopause [Rezeau et al., 1993; Song, 1994; Song et al., 1993; Phan and Paschmann, 1996, and references therein].

A good example of such a transition is illustrated by the WIND crossing of the magnetopause on December 12, 1996 which is shown in Fig. 1. In this figure, WIND is passing from the magnetosheath into the magnetosphere on the flank of the magnetosphere at roughly $X_{g s e}=0, Y_{g s e}=15 R_{E}, Z_{g s e}=0$. The 
background magnetic field undergoes a rotation of approximately $80^{\circ}$ during the crossing of the magnetopause. While in the magnetosheath (prior to 19:30 UT), the wave power is mainly in the compressional component as evidenced in the large fluctuations of $B_{t o t}$. The plasma $\beta$ for this period is moderate (around 2) and the pressure anisotropy is not very large [see Fig. 2 from Phan et al., 1997], suggestive that they are compressional Alfvén waves rather than mirror modes. The compressional wave activity continues through the magnetopause even though the plasma $\beta$ drops below unity in a plasma depletion layer (between 19:25 and 20:00 UT). As the density gradually decreases and the magnetic field increases, the transverse component of the magnetic field fluctuations becomes dominant, although the frequency range remains roughly the same. There is a marked increase in wave activity in the individual GSE Cartesian components of the magnetic field, but the fluctuations of $B_{t o t}$ remain roughly the same. The enhanced transverse wave activity persists all the way into the low-latitude boundary layer from (20:00 UT to 20:45 UT). This magnetopause crossing is a typical example of situations where compressional wave activity is found in the magnetosheath.

The change in wave polarization from the magnetosheath to the magnetosphere is clearly seen in the power spectrogram for the crossing. The spectrum is computed in a sliding Hanning window every 30 seconds and includes 4 minutes of data. The power spectral densities are obtained consistently using either the Fourier Transform or the Thompson Multitaper Method (with 128 modes). Power spectra are obtained for all magnetic field components and the magnetic field magnitude and are used to obtain the compressional and transverse magnetic field spectrum. In Fig. 2 we show the wave power spectral density of compressional, $P_{\|}$, and transverse, $P_{\perp}$, magnetic fluctuations as well as the fraction of wave power in the transverse magnetic field component. A broadband of waves is found in the $10-100 \mathrm{mHz}$ frequency range. In the magnetosheath, the waves are primarily compressional, but at the magnetopause, where the density and magnetic field gradients are found, the transverse component is dominant in the same frequency range. The compressional magnetic field spectra remain approximately the same from the magnetosheath into the magnetopause with an eventual cutoff as WIND moved through the low-latitude boundary layer into the plasma sheet at 20:30 UT. In the magnetosheath, the transverse component ap-

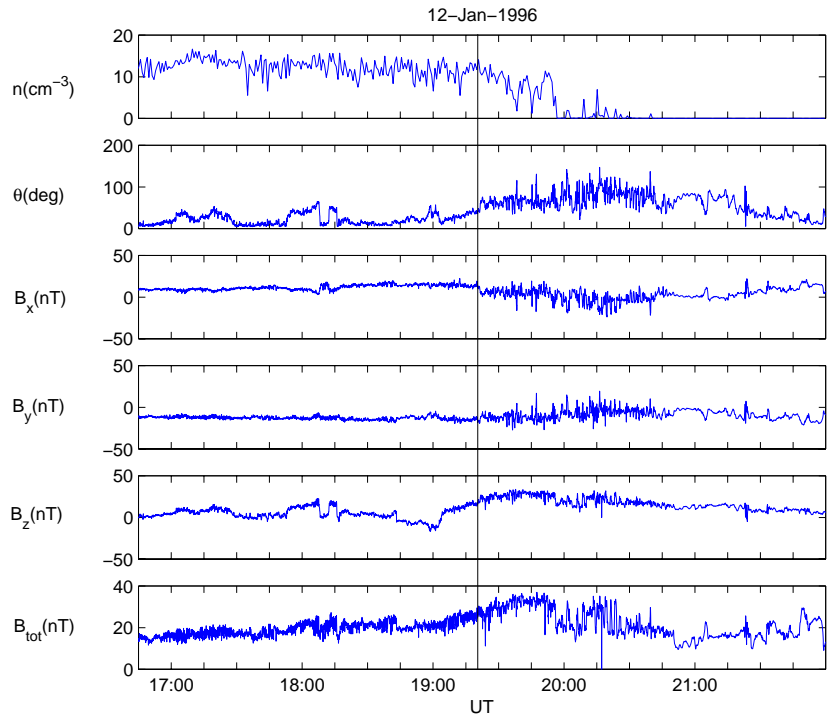

Figure 1. Density and magnetic field data from a magnetopause crossing by the WIND satellite on Jan 12, 1996 at approximately 19:30 UT as indicated by the vertical reference line. Note that in the magnetosheath (prior to 19:30 UT) wave power is primarily compressional. Coincident with the strong density gradients at the magnetopause there is a strong enhancement in the transverse components of the magnetic field fluctuations, but the compressional amplitude remains approximately the same as in the magnetosheath. The magnetic field angle relative to the sheath field rotates by about $\theta_{s h}=80^{\circ}$ during the crossing. Data courtesy of R. Lepping and R. Lin.

pears in the same frequency range and is well correlated with the compressional component but appears to fall off slightly faster with frequency suggestive that the wavevector is more oblique for higher frequency waves. However, at the magnetopause there is a dramatic increase in the transverse power spectrum as evidenced in the lower panel of Fig. 2. Notice also that the amplification (ratio of spectral density at the magnetopause to that of the magnetosheath) of the transverse spectrum falls off as frequency increases.

In order to understand the wave activity better, we have examined 13 cases of ISEE1, ISEE2, and WIND data as a function of magnetic shear, $\theta_{s h}$, which is defined as the angle between the magnetic field in the magnetosheath and the magnetic field on the magnetospheric side of the magnetopause. The crossings are listed in Table 1. In these cases compressional waves were found in the magnetosheath, the space- 


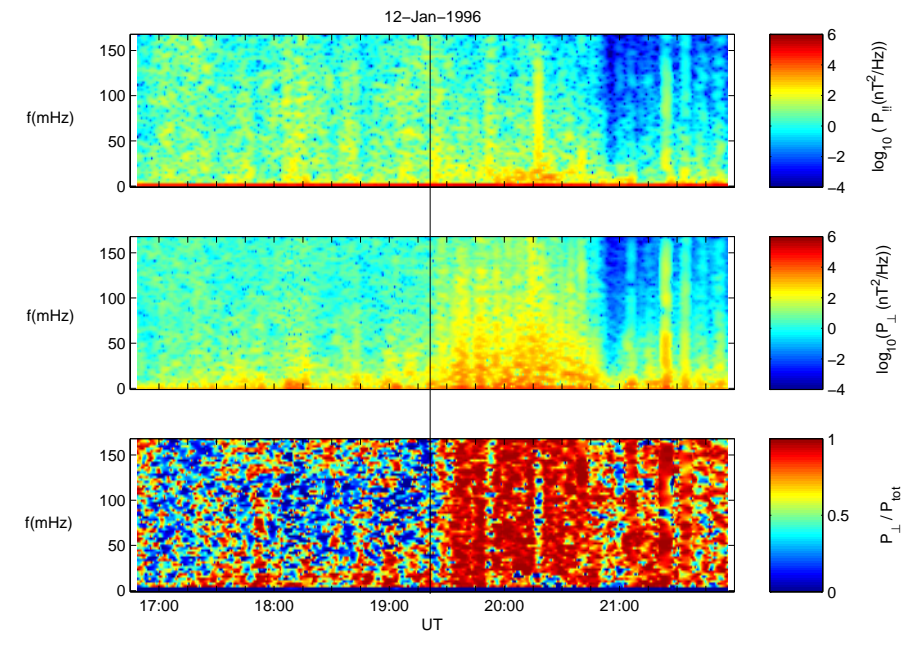

Figure 2. Wave power spectra for the WIND crossing of the magnetopause shown in Fig. 1. $P_{\|}, P_{\perp}$ and $P_{\text {tot }}$ are the power spectral densities obtained from $\left|\delta B_{\|}\right|^{2},\left|\delta B_{\perp}\right|^{2}$ and $|\delta \mathbf{B}|^{2}$ respectively. Notice that prior to crossing the magnetopause wave power is primarily compressional, coincident with the magnetopause crossing (indicated by the vertical line), the wave activity is primarily transverse. Note that the compressional wave component remains essentially the same before and during the magnetopause crossing.

craft remained at the magnetopause for an extended time, and the background density gradients were relatively smooth. The wave amplification $\left(P_{\perp m s h} / P_{\perp m p}\right.$ where $P_{\perp m s h}$ and $P_{\perp m p}$ refer to average values of the power spectral density of $\delta \mathbf{B}_{\perp}$ in the magnetosheath and magnetopause respectively) is shown in Fig. 3 for these magnetopause crossings for frequencies of 25 and $50 \mathrm{mHz}$. The results indicate: (1) The transverse wave component at the magnetopause is not significantly amplified below a threshold angle (approximately $60^{\circ}$ ), (2) Greatest amplification is for shear between $70^{\circ}$ and $180^{\circ}$, and (3) Waves with higher frequency are less amplified.

\section{Observations and Kinetic Alfvén Wave Theory}

These observations can be understood in the context of resonant mode conversion of compressional Alfvén waves into kinetic Alfvén waves at the magnetopause. Resonant mode conversion occurs when a compressional Alfvén wave propagates into a re-

\begin{tabular}{|c|c|c|}
\hline Spacecraft & Date & Shear Angle \\
\hline ISEE1 & $\mathbf{0 5 / 1 1 / 7 7}$ & $\mathbf{1 0}$ \\
\hline ISEE2 & $\mathbf{0 6 / 1 1} / 77$ & $\mathbf{1 4 0}$ \\
\hline ISEE2 & $\mathbf{2 4 / 1 1 / 7 7}$ & $\mathbf{5}$ \\
\hline ISEE2 & $\mathbf{2 9} / 11 / 77$ & $\mathbf{1 6 0}$ \\
\hline ISEE2 & $\mathbf{1 9} / \mathbf{1 7} / 78$ & $\mathbf{0}$ \\
\hline ISEE2 & $\mathbf{0 5} / 09 / 78$ & $\mathbf{9 0}$ \\
\hline ISEE1 & $\mathbf{0 1 / 1 1 / 7 8}$ & $\mathbf{5 0}$ \\
\hline ISEE1 & $\mathbf{2 5 / 1 1 / 7 8}$ & $\mathbf{1 3 0}$ \\
\hline ISEE1 & $\mathbf{2 1 / 1 0} / 79$ & $\mathbf{1 8 0}$ \\
\hline ISEE1 & $\mathbf{1 9} / 11 / 79$ & $\mathbf{8 0}$ \\
\hline ISEE1 & $\mathbf{1 9} / \mathbf{1 2} / 79$ & $\mathbf{1 7 0}$ \\
\hline ISEE1 & $\mathbf{2 3 / 1 2 / 7 9}$ & $\mathbf{7 0}$ \\
\hline WIND & $12 / 01 / 96$ & 80 \\
\hline
\end{tabular}

Table 1. Magnetopause Crossings

gion with gradients in $k_{\|} V_{A}$ such as at the magnetopause where the Alfvén velocity can increase by at least a factor of 10. At the magnetopause, gradients in the direction normal to the magnetopause boundary are dominant compared with gradients along the boundary, and we can approximate the background plasma and magnetic field profiles as functions of the coordinate, $x$, in the direction normal to the magnetopause. We assume the magnetic field is of the form $\mathbf{B}=B_{0}(x) \mathbf{b}$ where $\mathbf{b}=\cos \theta_{b}(x) \hat{\mathbf{z}}+\sin \theta_{b}(x) \hat{\mathbf{y}}$ and the magnetic field angle, $\theta_{b}$ rotates by an angle $\theta_{s h}$ across the magnetopause. The equilibrium profiles vary smoothly across the magnetopause on a scale of 10 ion gyroradii $\left(\rho_{i}\right)$. For such a configuration, wave propagation is well-described by the kinetic-fluid model [Cheng and Johnson, 1999] which simplifies to the following set of dimensionless coupled equations for $\mathcal{W}_{\|}=\delta p+B_{0} \delta B_{\|}$and $\mathcal{W}_{x}=i B_{0} \delta B_{x}$.

$$
\begin{gathered}
\frac{d^{2} \mathcal{W}_{\|}}{d x^{2}}=-\left(k_{A}^{2}-k_{S}^{2}\right) \mathcal{W}_{\|}+\left(k_{A}^{2}-k_{\|}^{2}\right) \delta p \\
+k_{\|}\left[\frac{d}{d x} \log \left(\frac{k_{\|}^{2}}{k_{A}^{2}}\right)\right] \mathcal{W}_{x} \\
\mathcal{K} \mathcal{W}_{x} \equiv\left[1+\frac{T_{e}}{T_{i}}\left(1+\hat{\eta} \frac{Z_{i}^{\prime}}{2}\right)\left(\frac{-2}{Z_{e}^{\prime}}\right)\right] k_{\|}^{2} \rho_{i}^{2} \frac{d^{2} \mathcal{W}_{x}}{d x^{2}} \\
=\left(k_{\|}^{2}-k_{A}^{2}\right) \mathcal{W}_{x}-k_{\|} \frac{d \mathcal{W}_{\|}}{d x} \\
\hat{\eta} \mathcal{W}_{x} \approx \int d x^{\prime} \frac{d k_{x}}{2 \pi} e^{i k_{x}\left(x-x^{\prime}\right)} b \frac{\left(\Gamma_{0}(b)-\Gamma_{1}(b)\right)}{\left(1-\Gamma_{0}(b)\right)} \mathcal{W}_{x}\left(x^{\prime}\right)
\end{gathered}
$$




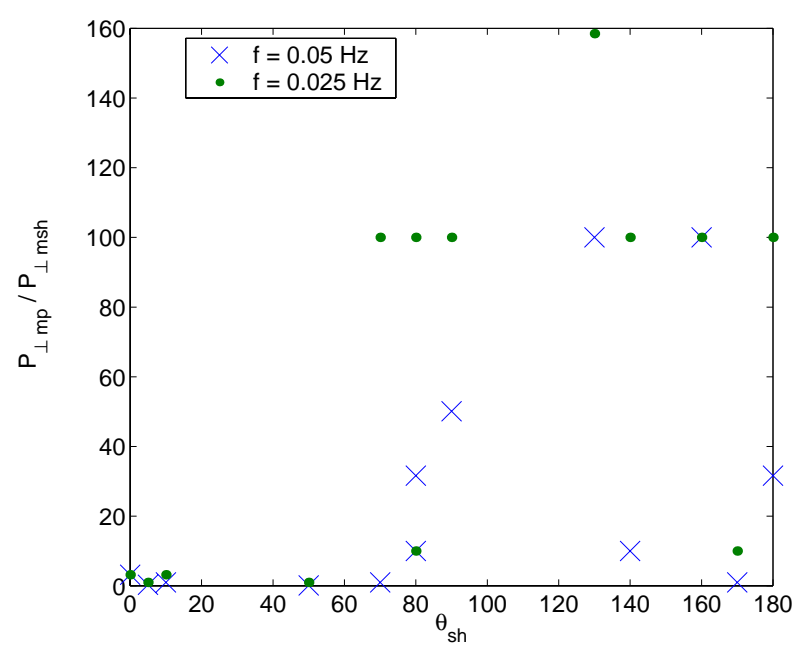

Figure 3. Amplification of waves at the magnetopause as a function of magnetic shear across the magnetopause boundary. The amplification is defined to be $P_{\perp m s h}(f) / P_{\perp m p}$ where the subscripts $m p$ and msh refer to the magnetopause and magnetosheath respectively. Data points are taken from the magnetopause crossings listed in Table 1.

$\mathbf{k}_{S}$ is the wavevector in the plane perpendicular to $x$; $k_{\|}=\mathbf{k} \cdot \mathbf{b}=\mathbf{k}_{S} \cdot \mathbf{b}=k_{S} \cos \theta_{s b}$, where $\theta_{s b}$ is the angle between $\mathbf{b}$ and $\mathbf{k}_{S}$; and $k_{A}^{2}=\omega^{2} / V_{A}^{2}$ is the Alfvén wavevector where $\omega$ is the wave frequency and $V_{A}$ is the Alfvén velocity. $Z_{s}^{\prime}$ is the derivative of the plasma dispersion function of argument $\zeta_{s}=\omega / \sqrt{2} k_{\|} v_{t s}$ for species $s$ with thermal velocity $v_{t s}$. We have taken the plasma to be isotropic. The operator $\hat{\eta}$ is a weakly nonlocal operator introduced by finite Larmor radius effects. The integration involves $\Gamma_{n}(b) \equiv I_{n}(b) e^{-b}$ where $b \equiv\left(k_{x}^{2}+k_{S}^{2} \sin ^{2} \theta_{b}\right) \rho_{i}^{2}$. Near the location where $k_{\|}^{2}=k_{A}^{2}, \hat{\eta} \approx 1+\mathcal{O}\left(\rho_{i}^{2} d^{2} / d x^{2}\right)$ and for $k_{A}^{2} \gg k_{\|}^{2}$ (as occurs when $k_{\|}$is small), the contribution of $\hat{\eta}$ vanishes.

The pressure equation required for the compressional wave is, $\delta p \approx(1-1 / \tau) \mathcal{W}_{\|}$where $\tau=1+$ $\sum_{s} \beta_{s}\left(1+Z_{s}^{\prime} / 2\right)$ and summation is over all species, $s$. The function $1 / \tau$ is well behaved for the frequencies of interest (in contrast to the MHD approach which gives a singularity where $\omega^{2}=k_{\|}^{2} C_{S}^{2} /(1+\beta)$. In a cold, isotropic plasma $\zeta_{i} \gg 1, \tau \rightarrow 1$ and there is no contribution from this term. In a warm plasma with $\zeta \sim 1, \tau \sim \mathcal{O}(1)$ and this term only introduces weak damping to the compressional wave [Johnson and Cheng, 1997a], so the sound resonance is not very important. Moreover, near the the Alfvén resonance where $k_{\|}^{2}=k_{A}^{2}$, the pressure term vanishes from Eq. 1 . Therefore, it is also not critical to include Larmor radius corrections in the term proportional to $\delta p$ in Eqs. 1 when describing wave behavior near the Alfvén resonance.

Note that Eqs. 1 and 2 do not explicitly involve the magnetic field component in the $\mathbf{b} \times \hat{\mathbf{x}}$ direction. Therefore these two equations along with appropriate boundary conditions determines $\mathcal{W}_{\|}$and $\mathcal{W}_{x}$. In the absence of kinetic effects $(\mathcal{K} \rightarrow 0)$, the singularities of Eqs. 1 and 2 occur where (1) $\delta p=-B_{0} \delta B_{\|}(\tau=$ $0)$ which corresponds to the slow mode resonance in MHD and (2) $\omega^{2}=k_{\|}^{2} V_{A}^{2}\left(\right.$ at $\left.k_{A}^{2}=k_{S}^{2} \cos ^{2} \theta_{k b}\right)$ which is the Alfvén resonance where the response of $\mathcal{W}_{x}$ is singular as found in Eq. 2. At the magnetopause the singularities are resolved by including kinetic effects.

Generally, the Alfvén velocity increases across the magnetopause from the magnetosheath side so that $k_{A}^{2}$ is a monotonically decreasing function. In the magnetosheath the wave is propagating which requires $k_{A}^{2}>k_{S}^{2}$. As the wave propagates across the magnetopause, $k_{A}$ decreases until $k_{A}^{2}=k_{S}^{2}$ where compressional wave is cutoff. Beyond that location, the compressional wave decays. However, at the location $k_{A}^{2}=k_{\|}^{2}$ the decaying compressional wave encounters the Alfvén resonance where it can be be reflected out of phase from the incoming wave. Near the resonance location the parallel magnetic field is well behaved, but the transverse fields are singular.

Ion gyroradius effects resolve the singular behavior and are described by the kinetic response operator, $\mathcal{K}$, which is an integral operator obtained in a straightforward way from [Cheng and Johnson, 1999]. The operator is obtained using a Padé approximate (valid for both large and small $k_{\perp}^{2} \rho_{i}^{2}$ ). We have also included the effects of the parallel electric field through the quasineutrality condition. In the limit $v_{i} \ll \omega / k_{\|} \ll v_{e}$, the usual KAW operator $\mathcal{K} \rightarrow\left(1+T_{e} / T_{i}\right) k_{\|}^{2} \rho_{i}^{2} d^{2} / d x^{2}$ is recovered (the Padé approximation accounts for the factor of 1 rather than $3 / 4)$. On the other hand, near the location where $k_{\|} \rightarrow 0, \omega / k_{\|} \gg v_{e}, v_{i}, \mathcal{K} \rightarrow-k_{A}^{2} \lambda_{e}^{2} d^{2} / d x^{2}$ where $\lambda_{e}=c / \omega_{p e}$. Note the change of sign in the coefficient of the operator indicates that the wave enters the inertial regime where it decays on the scale of the electron skin depth [Johnson and Cheng, 1997b; Cheng and Johnson, 1999].

Away from the Alfvén resonance, the waves described by Eqs. 1 and 2 are basically decoupled on two different scales: (1) the long MHD wavelength scale of the compressional wave, $k_{A}$, and (2) the short scale 
of the kinetic Alfvén wave, $\rho_{i}$. In the long wavelength limit, the transverse component obeys the simple relation $\mathcal{W}_{x}=k_{\|}\left(d \mathcal{W}_{\|} / d x\right) /\left(k_{\|}^{2}-k_{A}^{2}\right)$. In the short wavelength limit, Eq. 1 requires that $\mathcal{W}_{\|}$vanishes because the derivative term cannot be balanced by the other terms. For this reason, $\mathcal{W}_{\|}$consists almost entirely of the long wavelength compressional wave which is well behaved (constant) near the resonance. Hence, magnetic signatures in the compressional component are not significantly amplified while the transverse component is significantly amplified. Note that this property is nicely shown in Fig. 2 where the compressional wave power hardly changes in contrast to the transverse wave power. Near the Alfvén resonance it is necessary to solve the coupled system of equations.

We solve Eqs. 1 and 2 numerically on a nonuniform discrete grid and obtain the solutions through matrix manipulation. Boundary conditions are imposed at the magnetosheath and magnetosphere boundaries. The boundary condition in the magnetosheath is an incoming compressional MHD wave. At the magnetosphere boundary, the compressional MHD wave is decaying. For the kinetic Alfvén wave only radiating/decaying solutions are allowed. Boundary conditions are imposed asymptotically. The Alfven velocity is taken to increase by a factor of 10 across the magnetopause and the magnetic field rotates through an angle, $\theta_{s h}$.

A good measure of the efficiency of mode conversion at the magnetopause is the amount of compressional wave absorption in the magnetopause layer. Energy absorption is determined by comparing the Poynting flux $(\delta \mathbf{E} \times \delta \mathbf{B} \cdot \hat{\mathbf{x}})$ of the incident compressional wave $\left(S_{I}\right)$ with the Poynting flux of the reflected $\left(S_{R}\right)$ and transmitted $\left(S_{T}\right)$ compressional waves. The Poynting flux of the kinetic Alfvén wave in the magnetopause near the mode conversion layer is $S_{K A W}=S_{I}+S_{R}-S_{T}$. In the magnetopause, the transverse magnetic field component is mainly from the kinetic Alfvén wave, therefore $S_{K A W} \sim P_{\perp m p}$, and the compressional wave absorption, $A \equiv\left(S_{I}+S_{R}-S_{T}\right) / S_{I}$ is proportional to the wave amplification, $P_{\perp m p} / P_{\perp m s h}$. Depending on the profiles of $V_{A}$ and $\mathbf{k}_{S} \cdot \mathbf{b}$, there can be up to three resonance locations in the magnetopause. The absorbed energy is converted to kinetic Alfvén waves which: (a) propagate back into the magnetosheath (for a single resonance location), (b) propagate into both magnetosheath and magnetosphere (for two resonance locations), or (c) couple to a quasi-trapped kinetic Alfvén wave (for three resonance locations).
In order to obtain the total absorption as a function of frequency and magnetic shear, we must sum the absorption over the wavevector spectrum of incoming compressional waves. To do this, we assume that all wavevectors lie on a dispersion surface in wavevector space defined by $\omega(\mathbf{k})=$ const and integrate over the dispersion surface. We integrate over the angles of $\mathbf{k}: \theta_{k 0}$ is the angle between $k$ and the magnetic field in the magnetosheath and $\phi_{k 0}$ is the azimuthal angle in planes perpendicular to the magnetosheath magnetic field, $\mathbf{B}_{m s h}$. For compressional waves the dispersion surface is approximately defined by $\omega^{2} \approx k^{2}\left(V_{A}^{2}+C_{s}^{2} \sin ^{2} \theta_{k 0}\right)$. The wave vectors are approximately distributed on an ellipsoid with major radius $k=k_{A}$ and minor radii $k=k_{A} / \sqrt{1+C_{s}^{2} / V_{A}^{2}}$. The absorption spectrum as a function of frequency is obtained by integrating over the ellipsoid - that is, over the angles $\left(\theta_{k 0}, \phi_{k 0}\right)$ with $k^{2} \approx k_{A}^{2} /\left(1+C_{s}^{2} \sin ^{2} \theta_{k 0} / V_{A}^{2}\right)$ imposed by the dispersion relation. Moreover, compressional waves typically have $k_{\perp} \gg k_{\|}$so it is reasonable to assume that the spectrum is highly peaked around $\theta_{k 0}=\pi / 2$. On the other hand, there is no compelling reason to expect that the initial wave spectrum depends on the direction $\phi_{k 0}$, therefore we take a uniform weighting.

The wave observations show a strong dependence of amplification $\left(P_{\perp m p} / P_{\perp m s h}\right)$ on the shear angle across the magnetopause. For small shear angles, there is little wave amplification, while above a threshold amplification is enhanced and relatively level. The minimum in amplification for small shear is consistent with the mode conversion mechanism because the waves in the magnetosheath propagate nearly perpendicular to the magnetic field. The absorption coefficient, $A\left(\omega, \theta_{s h}\right)$ is presented in Figure 4. The absorption is obtained by computing the absorption coefficient as a function of $\left(\omega, \theta_{s h}, \theta_{k 0}, \phi_{k 0}\right)$ and performing an integration over the variables $\left(\theta_{k 0}, \phi_{k 0}\right)$ with uniform weight in $\phi_{k 0}$ and a strongly peaked weighting function about $\theta_{k 0}=\pi / 2$.

The absorption is the result of mode conversion to kinetic Alfvén waves and measures the efficiency of the mode conversion mechanism. The absorbed energy is the Poynting flux of the kinetic Alfvén wave which radiates away from the mode conversion location. The Poynting fluxes scale as the group velocity multiplied by spectral density. Because the kinetic Alfvén wave radiates slowly across the magnetic field, its amplitude must be greatly increased compared with the amplitude of the incoming MHD wave in order to carry away the mode converted energy from 
the field line resonance location. For kinetic Alfvén waves the Poynting flux is approximately, $S_{K A W} \sim$ $\left(\omega / k_{x}\right) k_{x}^{2} \rho^{2} /\left(1+k_{x}^{2} \rho^{2}\right) P_{\perp}$, while for the MHD wave $S_{M H D} \sim V_{A} P_{\perp}$. The kinetic Alfvén wavevector, $k_{x}$ can be estimated from dominant balance in Eq. 2$k_{x} \sim\left(\rho_{i}^{2} L\right)^{-1 / 3}$ where $L$ is the scale length of the Alfvén velocity gradient at the magnetopause. One can then estimate from the linear dispersion relation that the wave amplification $P_{\perp K A W} / P_{\perp M H D} \sim$ $A\left(1+(\rho / L)^{2 / 3}\right) V_{A} /\left[2 \pi f \rho(\rho / L)^{1 / 3}\right]$. For typical magnetopause parameters: $V_{A} \sim 300 \mathrm{~km} / \mathrm{s}, \rho_{i} \sim 50 \mathrm{~km}$, $L \sim 500 \mathrm{~km}, f=25 \mathrm{mHz}, P_{\perp K A W} / P_{\perp M H D} \sim 100 A$. As shown in the paper by Johnson and Cheng [1997b], amplification can also be larger near the Alfvén resonance depending on a number of other factors.

Because $\left|\delta \mathbf{B}_{\perp}^{2}\right|$ amplification scales directly with compressional wave absorption, the results of Figure 4 can be compared qualitatively with observed $\left|\delta \mathbf{B}_{\perp}^{2}\right|$ amplification. The important features to notice are: (1) for angles greater than $50^{\circ}$ the absorption is approximately constant, but for smaller shear there is a trough in $A$ and (2) the absorption decreases weakly as frequency increases for angles larger than $50^{\circ}$. However, for angles less than $50^{\circ}$ there is a significant broadening of the trough for higher frequency with far less absorption. These qualitative properties correspond well to observations of $\left|\delta \mathbf{B}_{\perp}^{2}\right|$ amplification as a function of magnetic shear angle and frequency as discussed in Fig. 3. The quantitative differences between the theory and data (for example, the theoretical threshold angle is smaller) can be attributed to the uncertainly involved in analyzing the data and the simplifications of the theoretical model.

\section{Discussion and Summary}

In this work we have examined the dependence of amplification of the transverse magnetic field component at the magnetopause as a function of magnetic shear angle across the magnetopause. We have analyzed a set of observational events which are typical of magnetopause crossings when strong compressional wave activity is found in the magnetosheath. Those events suggest that transverse wave amplification at the magnetopause is not significant up to a threshold angle around 60 degrees. Above this angle significant amplification results. Waves with higher frequency have less amplification of the transverse magnetic field component and exhibit a wider trough below the threshold. While there was significant amplification of the transverse magnetic power spectra, there
A

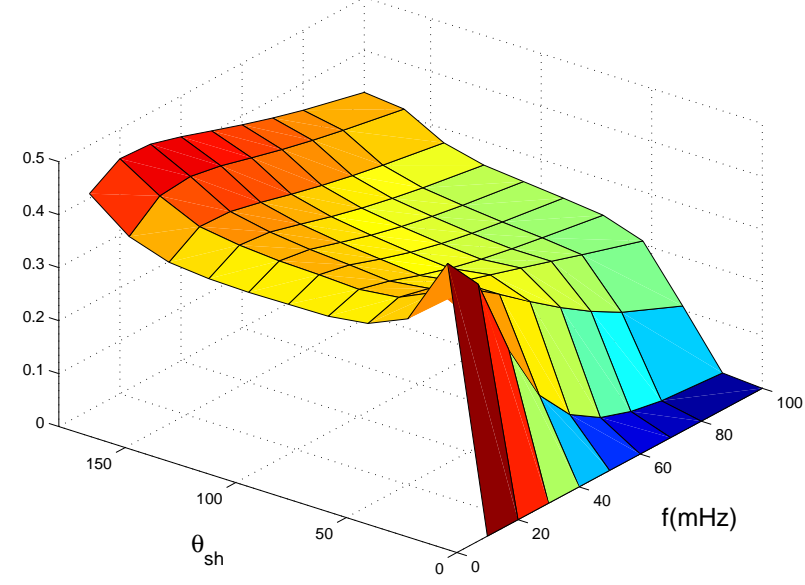

Figure 4. Absorption coefficient as a function of frequency and magnetic shear angle $\theta_{s h}$ (degrees).

was little enhancement of the compressional spectra.

We compared these observations with a theoretical calculation of compressional wave absorption via mode conversion into kinetic Alfvén waves at the magnetopause which has been proposed to be responsible for amplification of the transverse magnetic power spectra. We examined wave absorption as a function of frequency and magnetic shear angle. We integrated over the wavevector spectrum assuming that the incoming wave spectrum is strongly peaked with wavevector perpendicular to the magnetic field in the magnetosheath. The resulting absorption curve suggests that maximum absorption occurs at magnetic shear angles greater than approximately 50 degrees. For smaller angles, a trough in wave absorption is found which is broader for larger frequency. The wave absorption is a decreasing function of frequency for frequencies of interest. These properties are qualitatively consistent with the wave observations. Finally, the mode conversion process does not amplify the compressional magnetic field component consistent with observation.

These results imply that mode conversion of compressional MHD waves at the magnetopause to kinetic Alfvén waves is a real process. Moreover, based on previous studies the kinetic Alfvén waves are expected to provide significant particle transport and plasma heating at the magnetopause [Hasegawa and Mima, 1978; Johnson and Cheng, 1997b; Chen, 1999]. 
Acknowledgments We thank Paul Song for providing and identifying relevant ISEE1 and ISEE2 data and Dave Sibeck for useful discussions. This work is supported by NSF grant ATM-9523331.

\section{References}

Belmont, G., F. Reberac, and L. Rezeau, Resonant amplification of magnetosheath MHD fluctuations at the magnetopause, Geophys. Res. Lett., 22, 295298, 1995.

Chen, L., Theory of plasma transport induced by lowfrequency hydromagnetic waves, J. Geophys. Res., 104, 2421-2428, 1999.

Cheng, C. Z., and J. R. Johnson, A kinetic-fluid model, J. Geophys. Res., 104, 413-427, 1999.

Hasegawa, A., and K. Mima, Anomalous transport produced by kinetic Alfvén wave turbulence, $J$. Geophys. Res., 83, 1117-1123, 1978.

Johnson, J. R., and C. Z. Cheng, Global structure of mirror modes in the magnetosheath, J. Geophys. Res., 102, 7179-7189, 1997a.

Johnson, J. R., and C. Z. Cheng, Kinetic Alfvén waves and plasma transport at the magnetopause, Geophys. Res. Lett., 24, 1423-1426, 1997b.

Lee, L. C., J. R. Johnson, and Z. W. Ma, Kinetic Alfvén waves as a source of plasma transport at the dayside magnetopause, J. Geophys. Res., 99, 17405-17411, 1994.
Phan, T. D., and G. Paschmann, Low-latitude dayside magnetopause and boundary layer for high magnetic shear 1. structure and motion, J. Geophys. Res., 101, 1996.

Phan, T. D., et al., Low-latitude dusk flank magnetosheath, magnetopause, and boundary layer for low magnetic shear: Wind observations, J. Geophys. Res., 102, 1997.

Rezeau, L., A. Roux, and C. T. Russell, Characterization of small-scale structures at the magnetopause from ISEE measurements, J. Geophys. Res., 98, 179, 1993.

Song, P., Observations of waves at the dayside magnetopause, in Solar Wind Source of Magnetospheric Ultra-Low-Frequency Waves, Geophysical Monograph Series, vol. 81, pp. 159-171. 1994.

Song, P., C. T. Russell, R. J. Strangeway, J. R. Wygant, C. A. Cattell, R. J. Fitzenreiter, and R. R. Anderson, Wave properties near the subsolar magnetopause: Pc 3-4 energy coupling for northward interplanetary magnetic field, J. Geophys. Res., 98, 187-196, 1993.

This preprint was prepared with the AGU LATEX macros v3.0. File GRLpp formatted 2000 July 19.

With the extension package ' $\mathrm{AGU}^{++}$', version 1.2 from $1995 / 01 / 12$ 
The Princeton Plasma Physics Laboratory is operated by Princeton University under contract with the U.S. Department of Energy.

\author{
Information Services \\ Princeton Plasma Physics Laboratory \\ P.O. Box 451 \\ Princeton, NJ 08543
}

Phone: 609-243-2750

Fax: 609-243-2751

e-mail: pppl_info@pppl.gov

Internet Address: http://www.pppl.gov 